\title{
EDITORIAL
}

\section{El envenenamiento infantil por plomo: un problema de años que demanda soluciones nuevas}

\section{$\mathrm{N}$ iños envenenados con plomo}

Los niveles de plomo extremadamente altos pueden ser devastadores y conducir al estado de coma y a la muerte. Inclusive niveles más moderados de plomo pueden ocasionar un efecto negativo a largo plazo sobre el desarrollo neurocognoscitivo de los niños. Estos hechos se conocen desde hace décadas.

Recientemente, sin embargo, la investigación científica señaló que el plomo afecta a los niños en niveles más bajos de los considerados anteriormente. Los resultados de los estudios indican que el plomo, aun en niveles inferiores a $10 \mu \mathrm{g} / \mathrm{dl}$, puede estar asociado con efectos adversos sobre el neurocomportamiento y el desarrollo cognoscitivos en los niños. ${ }^{1}$

Las buenas noticias en los Estados Unidos de América (EUA) son que, durante los últimos 25 años, los niveles de plomo en los niños han disminuido de manera drástica. Los datos del Tercer Estudio Nacional de Investigación sobre la Salud y la Nutrición, de los Centros de Control de las Enfermedades (CDC's NHANES III, por sus siglas en inglés), Fase 2 (19911994), mostró que el promedio de los niveles de plomo en la sangre, en niños de cinco años de edad o menores, ha disminuido en aproximadamente $80 \%$ desde la década de los $70 .{ }^{2}$ Los datos de la NHANES muestran una continuidad de esta tendencia, con una media geométrica que disminuyó de $2.7 \mu \mathrm{g} / \mathrm{dl}$ en el NHANES III, hasta $2.0 \mu \mathrm{g} / \mathrm{dl}$, en $1999 .{ }^{3}$ Este éxito en la reducción de los niveles de plomo en los niños de los EUA se encuentra asociado directamente con la implantación de intervenciones en el medio ambiente, de las cuales la más importante fue la eliminación del plomo en la gasolina.

Aunque en general los niveles de plomo en la sangre han disminuido, el problema del envenenamiento por plomo en los EUA no se ha resuelto. Niveles elevados de plomo se mantienen concentrados en los niños pobres de las minorías y en vecindarios de construcciones antiguas. Los datos de la vigilancia epidemiológica mostraron que la prevalencia de los niveles elevados de plomo en la sangre (EBLLs, por sus siglas en inglés: Elevated Blood Levels) es 30 veces mayor en los niños de ingresos más bajos que habitan construcciones viejas, que en niños de clase media que habitan en residencias más nuevas. ${ }^{4}$

Se mostró que la eliminación del plomo en la gasolina reduce de manera drástica los niveles de plomo en niños, no solamente en los EUA sino también en muchos otros países del mundo. Sin embargo, los niños en América Latina y El Caribe se encuentran expuestos al plomo a través de otras vías. La manufactura y el reciclaje de baterías, las plantas de fundición, la industria minera, la cerámica vidriada con plomo y las prácticas medicinales culturales son sólo algunas fuentes de intoxicación por plomo que afectan a nuestros niños. Las intervenciones efectivas en la salud pública deben intentar dirigir, de manera comprehensiva, cualesquiera que sean las fuentes de plomo.

Aunque existen grandes avances en el conocimiento sobre los efectos del plomo en los niños, la comunidad de la salud pública no ha tenido el mismo éxito en la eliminación de esta amenaza para la salud y el desarrollo de nuestros niños. El envenenamiento infantil por plomo es una enfermedad prevenible y representa un reto importante para los programas de salud pública a lo largo de América Latina y El Caribe.

En el mes de mayo del año 2001, la Organización Panamericana de la Salud convocó al Taller Latinoamericano Envenenamiento Infantil por Plomo: Evaluación, Prevención y Tratamiento, en Lima, Perú. La finalidad de este taller fue reunir a los profesionales 
de la salud de América Latina y de la Región de El Caribe para discutir los aspectos del envenenamiento por plomo en sus respectivos países, y desarrollar las recomendaciones dirigidas a reducir los niveles de plomo en la Región. Esta publicación representa las actas de las sesiones de este taller y proporciona, además, un enfoque conciso de muchos de los aspectos específicos del envenenamiento por plomo en la Región. Las recomendaciones entregan una metodología realista para abordar con eficiencia el envenenamiento infantil por plomo.

La información de alta calidad proporciona el fundamento para todos los programas exitosos de salud pública. Mediante el uso de los datos establecidos se pueden diseñar y enfocar los esfuerzos de intervención sobre los problemas más apremiantes y para las poblaciones más afectadas. Este taller representa un primer paso importante en el control del envenenamiento por plomo, ya que proporciona la oportunidad para el intercambio y la discusión de la información específica de cada país sobre este problema. Espero que el presente número de Salud Pública de México pro- porcione el impulso para poner en marcha una respuesta comprehensiva dirigida a la eliminación del envenenamiento infantil por plomo, considerado como una amenaza para la salud pública en América Latina y la Región de El Caribe.

Michael A McGeehin, PhD, MSPH.*

\section{Referencias}

1. Achwartz J. Low-level lead exposure and children's IQ :A meta-analysis and search for a threshold. Environ 1994;65:42-55. 2. Centers for Disease Control and Prevention. U pdate: Blood lead levels- United States, 1991-1994. MMW R Morb Mortal W kly Rep 1997;46:141-146.

3. Centers for Disease Control and Prevention. Blood lead levels in young children-U nites States and selected states, 1996-1999. MMW R Morb Mortal W kly Rep 2000;49:1133-1137.

4. Pirkle JL, Kaufmann RB, Brody DJ, Hickman T, Gunter EW, Paschal DC. Exposure of the US population to lead, 1991-1994. Environ Health Perspect 1998;106:745-750.

\footnotetext{
* Director, Division of Environmental Hazards and Health Effects, National Center for Environmental Health, Centers for Disease Control and Prevention 1600 Clifton Road, N.E., Mail Stop E-19.
} 